\title{
A Model for Combustion of Firebrands of Various Shapes
}

\author{
HOWARD R. BAUM ${ }^{1}$ and ARVIND ATREYA ${ }^{2}$ \\ ${ }^{1}$ Department of Fire Protection Engineering \\ University of Maryland, College Park, MD 20742 \\ ${ }^{2}$ Department of Mechanical Engineering \\ University of Michigan, Ann Arbor, MI 48109
}

\begin{abstract}
The lifetime of a firebrand before burning out controls the maximum distance a firebrand can travel to cause spotting. Thus, combustion of firebrands of various shapes and sizes and their burnout time during transport is studied. The analysis assumes "quasi-steady" burning. In the present context, "quasi-steady" means that the rate processes controlling the gas phase fuel consumption and energy release are much faster than the particle fuel depletion time or the gas phase transport times. The Reynolds number based on the overall particle dimension and velocity relative to the particle is assumed to be small. The gas phase combustion processes are represented by the evolution of a mixture fraction variable. It is shown that the velocity field near the particle can be described by a potential flow whose functional form is determined by the mass conservation equation and that this flow satisfies the particle surface boundary conditions. Gas phase solutions are obtained for two-parameter family of firebrand shapes composed of oblate and prolate ellipsoids of revolution. Prolate ellipsoids range from a thin needle to a sphere and oblate ellipsoids range from a sphere to a thin disc. Thus, they cover all possible firebrand shapes. The ambient velocity field does not need to be aligned with the firebrand axis of symmetry, so that the composite velocity and mixture fraction fields are three-dimensional. While a variety of steady-state condensed phase models are compatible with this picture, results are first presented for an ablating solid describable by the Spalding B number. B-numbers representative of flaming combustion of wood firebrands and glowing combustion of remaining char are used. All quantities are calculated as a function of the ellipsoidal aspect ratio, B number, and the Reynolds number. Surprisingly, it is found that the firebrand burnout time is shape independent. All possible shapes were considered by using oblate and prolate ellipsoids of different sizes and aspect ratios. The burnout time depends only on the firebrand mass under the assumptions used.
\end{abstract}

KEYWORDS: wildfires, firebrands, combustion, modeling

\section{NOMENCLATURE}

$\begin{array}{llll}\boldsymbol{B} & \text { Spalding B number } & \text { Greek } & \\ \mathcal{D} & \text { mass diffusivity }\left(\mathrm{m}^{2} / \mathrm{s}\right) & (\xi, \eta) & \text { ellipsoidal coordinates } \\ \Delta H & \text { energy released } / \text { mass of } \mathrm{O}_{2}(\mathrm{~kJ} / \mathrm{kg}) & \phi & \text { velocity potential } \\ L & \text { particle dimension }(\mathrm{m}) & \xi_{o} & \text { particle surface, } Z\left(\xi_{O}\right)=1 \\ \mathcal{L} & \text { heat of pyrolysis }(\mathrm{kJ} / \mathrm{kg}) & \epsilon & \text { aspect ratio: minor/major axis } \\ \dot{M} & \text { total mass rate leaving the particle } & \text { subscrip } & \\ S & \text { stoichiometric ratio }=v_{O} m_{O} / v_{F} m_{F} & \infty & \text { ambient conditions } \\ R e & \text { Reynolds number } & F & \text { fuel } \\ \tilde{T}_{\infty} & \text { ambient temperature }(\mathrm{K}) & O & \text { oxygen } \\ \tilde{T}_{w} & \text { particle surface temperature }(\mathrm{K}) & f l & \text { flame } \\ \vec{u} & \text { velocity vector }(\mathrm{m} / \mathrm{s}) & \text { superscripts } \\ \vec{U}_{\infty} & \text { relative velocity }(\mathrm{m} / \mathrm{s}) & \infty & \text { ambient conditions } \\ Y & \text { mass fraction } & \text { accents } & \\ Z & \text { mixture fraction } & \sim & \text { dimensional quantity } \\ (\tilde{x}, \tilde{r}) & \text { cylindrical coordinates }(\mathrm{m}) & \rightarrow & \text { vector quantity }\end{array}$




\section{INTRODUCTION}

Spotting ignition by lofted firebrands is a major fire propagation mechanism in wildland and wildlandurban-interface fires. It consists of many complex individual phenomena: (i) Generation of various size and shape firebrands by fires, (ii) Firebrand lofting and transport by fire-induced flows and prevailing winds, (iii) Combustion of various size and shape firebrands during transport, and (iv) Ignition after the firebrand lands on the fuel bed. Clearly, a range of phenomena must be understood to predict spotting ignition. A substantial amount of work has been done to develop this understanding starting from the pioneering work of Tarifa et al. [1] and Albini [2] to the more recent work of Manzello et al. [3], Koo et al. [4], Woycheese et al. [5], Sardoy et al. [6] and others. An excellent review of this literature has been recently published by Koo et al. [7]. The present work limits itself to the combustion part of the problem. It develops an analytical model to determine the burning rate and burnout time of firebrands. A simple physically interesting case of an ablating solid describable by the Spalding B-number is considered first. Charring solids that pyrolyze and form char require a numerical solution and are left for later analysis. The problem is solved in prolate and oblate ellipsoidal coordinates to cover all possible shapes. Prolate ellipsoids include firebrands ranging from a needle to a sphere, whereas, oblate ellipsoids include firebrands ranging from a thin disk (leaf) to a sphere. The advantages of using these coordinates are: (i) A single combustion solution suffices for all sizes and shapes; (ii) It can be shown that the near-field fluid flow around these objects simplifies to a potential flow and can be represented as the gradient of the velocity potential $\tilde{\phi}$. The analysis also assumes that the particle motion relative to the oxidizing atmosphere is small, yielding small Reynolds numbers based on the particle dimension ' $L$ ' and the relative velocity $\vec{U}_{\infty}$. It is further assumed that combustion can be adequately represented by the evolution of a mixture fraction variable ' $Z$ '. Thus, the energy and species conservation equations reduce to a single evolution equation for the mixture fraction.

With these assumptions, the mass conservation equation and the mixture fraction evolution equation, supplemented by an isobaric equation of state and a set of piecewise linear algebraic relations between the major gas species, the sensible enthalpy, and the mixture fraction constitute the system of equations describing the gas phase phenomena. The assumption that the velocity field is irrotational replaces the momentum conservation equations. It may appear contradictory to describe a low Reynolds number flow as an irrotational flow, but that is precisely what is assumed in all analyses of the combustion of spherical droplets. In that case, the spherically symmetric geometry precludes the existence of vorticity. For more general shapes, if a solution to the equations can be found consistent with a constant value of the velocity potential on the surface of the particle, then the solution so obtained satisfies the no-slip conditions at the particle surface, while still allowing for the emission of fuel vapor from the surface. This is precisely the case for prolate and oblate solids of revolution. Examples of these solids are shown in Fig. 1. In addition to
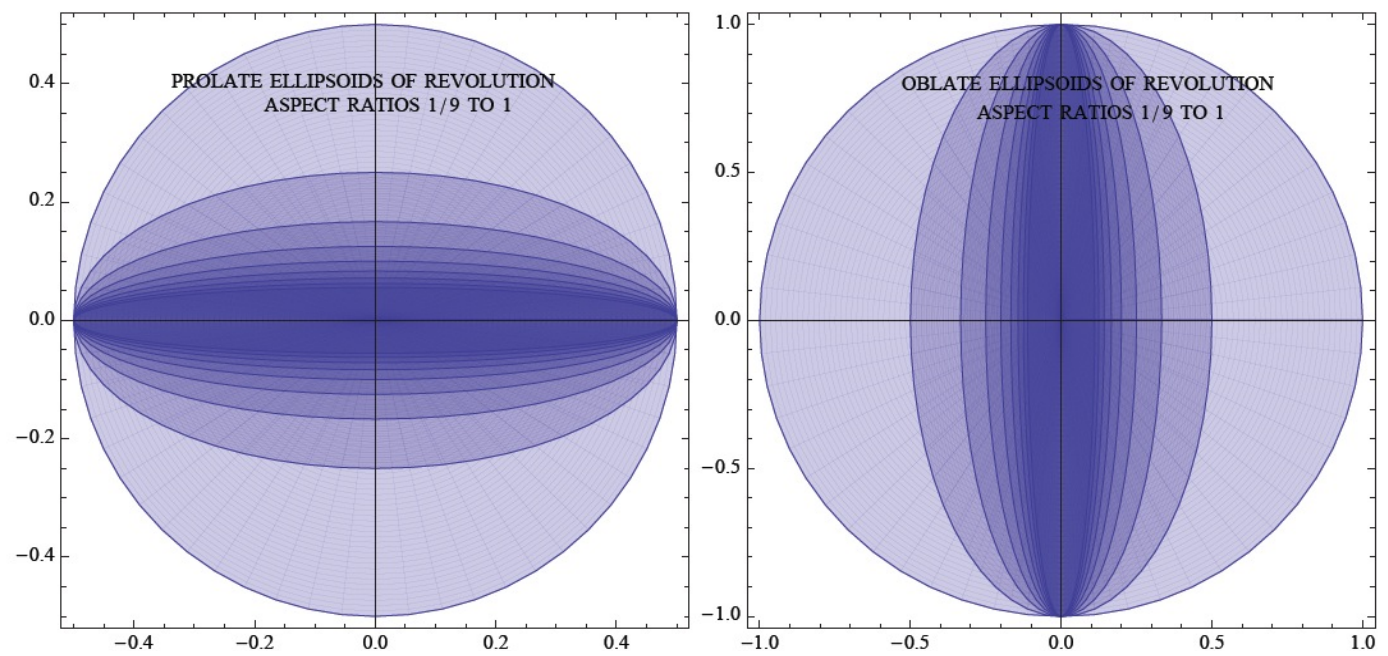

Fig. 1. Prolate Ellipsoids (left) and Oblate Ellipsoids (right) for various aspect ratios $\epsilon$. The limiting case of a Prolate Ellipsoid as $\epsilon \rightarrow 0$ is a needle while that for an Oblate Ellipsoid is a disc. 
the overall length $L$, a single parameter ' $\epsilon$ ' describing the ratio of semi-minor to semi-major axes lengths determines the specific geometry under consideration.

The remainder of this paper is organized as follows. First the models needed to describe phenomena in the gas phase are introduced. These models take different forms depending upon the distance from the particle for which solutions are required. Then, appropriate solutions for different parts of the domain of interest are developed but only near-field solutions are presented due to lack of space. Much of this can be done without considering anything beyond the geometry of the solid material. Following this, the model for an ablating solid is introduced and solved. This permits the gas phase solution to be completed for at least the simplest representation of the solid degradation. The ultimate result is a description of a set of timedependent processes that determine the burning rate of an idealized wood chip or wood char as a function of its size, shape, and material properties.

\section{GAS PHASE MODEL}

Assuming that for length scales of $O(L)$ from the particle, all processes can be regarded as quasi-steady, in the sense that the equations governing the gas phase phenomena can be utilized in their steady state form. The mass, energy, and species conservation equations then take the form:

$$
\begin{aligned}
& \nabla \cdot(\tilde{\rho} \vec{u})=0 \\
& \nabla \cdot(\tilde{\rho} \vec{u} Z)-\nabla \cdot(\tilde{\rho} \tilde{\mathcal{D}} \nabla Z)=0
\end{aligned}
$$

Here, $\tilde{\rho}(Z)$ is the gas density and $\tilde{\mathcal{D}}(Z)$ is the diffusivity.

The momentum equation can be formally solved by the assumption that the flow field on this length scale is irrotational. This can be shown by writing the momentum equations for a constant viscosity and assuming $\rho$ to be constant in the momentum equations only. Then if we require the vorticity to $\operatorname{vanish}(\vec{\omega}=0)$, we may write the momentum equations as [8]:

$$
\rho\left[\frac{\partial \vec{u}}{\partial t}+\nabla(\vec{u})^{2} / 2\right]+\nabla\left(\tilde{p}-\tilde{p}_{\infty}\right)=\frac{4}{3} \mu \nabla(\nabla \cdot \vec{u})
$$

Using $\vec{u}=\nabla \tilde{\phi}$, Eq. 3 can be immediately integrated subject to the boundary conditions that $\tilde{p}-\tilde{p}_{\infty}$ and $\vec{u}$ vanish far from the particle. The result is the generalized Bernoulli equation:

$$
\rho\left[\frac{\partial \tilde{\phi}}{\partial \tilde{t}}+(\vec{u})^{2} / 2\right]+\left(\tilde{p}-\tilde{p}_{\infty}\right)-\frac{4}{3} \mu \nabla \cdot \vec{u}=0
$$

The irrotational velocity field together with the generalized Bernoulli equation satisfies the no-slip boundary condition so long as the velocity potential is constant along the particle surface. Thus, the all solutions obtained below are exact solutions of the low Mach number combustion equations. The normal component of the velocity does not vanish and varies along the surface as a function of the size and shape of the particle.

Rewriting Eq. $1 \& 2$ in terms of the velocity potential $\vec{u}=\nabla \tilde{\phi}$, we get:

$$
\nabla \cdot(\tilde{\rho} \nabla \tilde{\phi})=0
$$


$\nabla \cdot(\tilde{\rho} Z \nabla \tilde{\phi})-\nabla \cdot(\tilde{\rho} \tilde{\mathcal{D}} \nabla Z)=0$

Once $\tilde{\rho}(Z)$ and $\tilde{\mathcal{D}}(Z)$ are specified, together with an appropriate set of boundary conditions, Eq. (5) and (6) are sufficient to determine the solution to the gas phase combustion problem in the vicinity of the particle. The diffusivity is specified by the relation:

$(\tilde{\rho})^{n} \tilde{\mathcal{D}}=\left(\tilde{\rho}_{\infty}\right)^{n} \tilde{\mathcal{D}}_{\infty}$

The value of $n$ will be chosen based on a balance between physical accuracy and mathematical convenience. Further, if the mean molecular weight at any point in the gas is assumed to be the same, rather than depending on the local species concentrations, the equation of state simplifies to:

$\tilde{\rho} \tilde{T}=\tilde{\rho}_{\infty} \tilde{T}_{\infty}$

More generally, the state equations now take the following form:

$$
\begin{aligned}
& Z=\left(S Y_{F}-\left(Y_{O}-Y_{O}^{\infty}\right)\right) /\left(S+Y_{O}^{\infty}\right) \\
& Z=\left((1+S) Y_{F}+Y_{P}\right) /(1+S) \\
& Z=\left(\Delta H\left(Y_{O}-Y_{O}^{\infty}\right)+C_{p}\left(\tilde{T}-\tilde{T}_{\infty}\right)\right) /\left(C_{p}\left(\tilde{T}_{w}-\tilde{T}_{\infty}\right)-\Delta H Y_{O}^{\infty}\right)
\end{aligned}
$$

Here, $\mathrm{S}$ denotes the mass of oxygen consumed per unit mass of fuel, $\Delta \mathrm{H}$ is the energy release per unit mass of oxygen consumed, $\tilde{T}_{w}$ is the temperature of the particle surface. The stoichiometry parameter $\mathrm{S}$ is another empirical input into this model. It can be used to characterize the consequences of incomplete combustion, although the procedure for choosing this parameter is beyond the present analysis.

Equations (9), (10), and (11) actually constitute several equations. Since we assume that the reactions are infinitely fast, the fuel and oxidizer cannot co-exist. Thus, the flame sheet is located on a curve $Z=Z_{f l}$, where the flame sheet value of $Z_{f l}$ is:

$$
Z_{f l}=Y_{O}^{\infty} /\left(S+Y_{O}^{\infty}\right)
$$

For values of $Z$ such that $Z \leq Z_{f l}, \mathrm{Y}_{\mathrm{F}}=0$ and Eq. 9 determines $Y_{O}(Z)$; while for $Z \geq Z_{f l}, \mathrm{Y}_{\mathrm{O}}=0$ and Eq. 9 determines $Y_{F}$. The combustion product mass fraction $Y_{P}$ is found from Eq.10 using the results for $Y_{F}$. Similarly, Eq. 11 determines the temperature $\tilde{T}(Z)$ as a piecewise linear function of $Z$. Eq. 8 then determines the piecewise linear relation $\tilde{\rho}(Z)$.

The boundary conditions on $Z$ are that $Z=0$ far from the particle and $Z=1$ on the particle surface. Similarly, the velocity potential $\phi$ is constant at the particle surface. Far from the surface:

$$
\tilde{\phi} \sim-\dot{M} /\left(4 \pi \tilde{\rho}_{\infty}|\vec{r}|\right)
$$


The quantity $\dot{M}$ is the total rate of mass leaving the particle surface, and $|\vec{r}|$ is the distance from the particle measured from its center of mass. The constancy of $\phi$ at the surface ensures that there is no slip at the surface, while the asymptotic condition guarantees that the total mass flux from the surface is preserved.

These conditions can be made more precise by the introduction of dimensionless prolate ellipsoidal coordinates [9]. First consider the prolate ellipsoid. Let $(\tilde{x}, \tilde{r})$ be the dimensional axial and radial coordinates in a cylindrical coordinate system aligned with the axis of symmetry of the ellipsoidal particle, arranged so that the particle occupies the domain defined by $-L / 2 \leq \tilde{x} \leq L / 2$. The prolate ellipsoidal coordinate system $(\xi, \eta)$ is then defined as follows:

$\tilde{r}^{2}=c^{2}\left(1-\eta^{2}\right)\left(\xi^{2}-1\right)$ and $\tilde{x}=c \xi \eta$

The quantity $c$ is defined by noting that $\xi=\xi_{0}$ gives a prolate ellipsoid of rotation with semi-major axis $a=$ $c \xi_{0}$ and semi-minor axis $b=c \sqrt{\left(\xi_{0}^{2}-1\right)}$. Thus:

$\frac{\tilde{x}^{2}}{c^{2} \xi_{0}^{2}}+\frac{\tilde{r}^{2}}{c^{2}\left(\xi_{0}^{2}-1\right)}=1$

Since the aspect ratio $\epsilon$ is the ratio of semi-minor to semi-major axes,

$e=c \sqrt{\xi_{0}^{2}-1} /\left(c \xi_{0}\right) \quad \xi_{0}=\left(\sqrt{1-e^{2}}\right)^{-1}$

Now using the fact that the semi-major axis has length $\mathrm{L} / 2$ :

$c=\frac{L}{2} \sqrt{1-e^{2}}$

Finally, the ellipsoidal coordinates can be written explicitly in terms of $(\tilde{x}, \tilde{r})$ as follows:

$$
\xi=\frac{1}{\sqrt{2} c}\left(\tilde{x}^{2}+\tilde{r}^{2}+c^{2}+\sqrt{\left(\tilde{x}^{2}+\tilde{r}^{2}+c^{2}\right)^{2}-(2 \tilde{x} c)^{2}}\right)^{1 / 2} \quad \eta=\frac{\tilde{x}}{c \xi}
$$

The coordinate geometry for contours of constant $\xi$ starting at the particle surface is shown for an ellipsoid of aspect ratio $\epsilon=1 / 4$ in Fig. 2 .

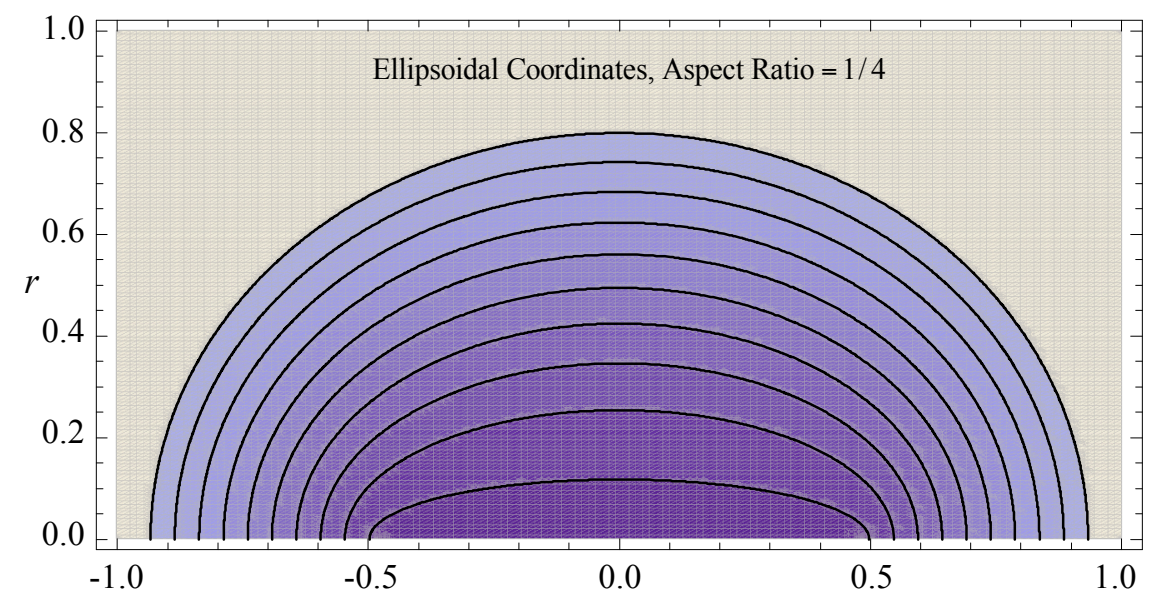




\section{NEAR FIELD SOLUTION}

Writing the conservation Eq. 1 and 2 in ellipsoidal coordinates and assuming that $\tilde{\rho}, \tilde{\phi}$ and $Z$ are functions of $\xi$ only, we get:

$\frac{d}{d \xi}\left(\tilde{\rho}(\xi)\left(\xi^{2}-1\right) \frac{d \tilde{\phi}}{d \xi}(\xi)\right)=0$

$\frac{d}{d \xi}\left(\tilde{\rho} Z(\xi)\left(\xi^{2}-1\right) \frac{d \tilde{\phi}}{d \xi}(\xi)\right)-\frac{d}{d \xi}\left(\tilde{\rho} \tilde{\mathcal{D}}(\xi)\left(\xi^{2}-1\right) \frac{d Z}{d \xi}(\xi)\right)=0$

Note that the assumption that $\tilde{\phi}$ is a function of $\xi$ does not mean that the velocity depends only on $\xi$, but only that the vectors are aligned in the direction of increasing $\xi$. However, since there is no component of the velocity parallel to any surface of constant $\xi$, the no slip condition at the particular surface $\xi=\xi_{0}$ is satisfied. The mass conservation Eq. 19 can now be readily integrated to yield:

$\tilde{\rho}(\xi)\left(\xi^{2}-1\right) \frac{d \tilde{\phi}}{d \xi}(\xi)=\tilde{M}$

Note that since $\tilde{\rho} \rightarrow \tilde{\rho}_{\infty}$ as $\xi \rightarrow \infty$, then the velocity vanishes far from the particle. Next, using equation (21), the energy and species conservation equation (20) can be integrated with the following result:

$$
\tilde{M} Z-\tilde{\rho} \tilde{\mathcal{D}}(\xi)\left(\xi^{2}-1\right) \frac{d Z}{d \xi}=\tilde{E}
$$

Integrating Eq. 22 and applying the boundary condition $\mathrm{Z}\left(\xi_{o}\right)=1$ we obtain:

$$
\frac{1}{2} \log \left(\frac{(\xi-1)\left(\xi_{0}+1\right)}{(\xi+1)\left(\xi_{0}-1\right)}\right)=\int_{Z}^{1} \frac{\tilde{\rho}(Z) \tilde{\mathcal{D}}(Z) d Z}{\tilde{E}-\tilde{M} Z}
$$

At this stage the constants of integration $\tilde{E}$ and $\tilde{M}$ are unknown. However, one relation between the two constants can be found by enforcing the boundary condition that $Z \rightarrow 0$ as $\xi \rightarrow \infty$. Thus the solution given by Eq. 23 can now be written as:

$$
\frac{1}{2} \log \left(\frac{(\xi+1)}{(\xi-1)}\right)=\int_{0}^{Z} \frac{\tilde{\rho}(Z) \tilde{\mathcal{D}}(Z) d Z}{\tilde{E}-\tilde{M} Z}
$$

Note that for $|\vec{r}| / L \gg 1, \xi \gg 1$ and $\xi \simeq|\vec{r}| / c$. Thus, far from the particle, the solution for $Z$ reduces to:

$$
Z \simeq \tilde{E} /\left(\tilde{\rho}_{\infty} \tilde{\mathcal{D}}_{\infty} \xi\right)=\tilde{E} c /\left(\tilde{\rho}_{\infty} \tilde{\mathcal{D}}_{\infty}|\vec{r}|\right)
$$


The solution becomes spherically symmetric far from the particle, regardless of its shape. This fact, which is a direct consequence of the ellipsoidal coordinate system, is used advantageously in the far field analysis. However, space does not permit presenting the details of the far field analysis. Thus, only a brief summary of the composite solution is presented.

\section{Composite Solution}

A composite solution for the Mixture Fraction that is accurate to the leading order in the small parameter $\delta=\vec{U}_{\infty} L / \tilde{\mathcal{D}}_{\infty}$ everywhere is found and it is presented below in Eq. 26.

$$
\frac{1}{2} \log \left(\frac{(\xi+1)}{(\xi-1)}\right) \exp ((\vec{s} \cdot \vec{y}-y) / 2)=\int_{0}^{Z} \frac{\rho(Z) \mathcal{D}(Z) d Z}{E-M Z}
$$

Here $\vec{s}$ is a the unit vector in the direction of $\vec{U}_{\infty}, \vec{y}=\delta \vec{r} / L, y=|\vec{y}|$ and the following non-dimensional definitions are used: $\tilde{\mathcal{D}}=\tilde{\mathcal{D}}_{\infty} \mathcal{D}(\vec{y}) ; \tilde{\rho}=\tilde{\rho}_{\infty} \rho(\vec{y}) ; \tilde{E}=\tilde{\rho}_{\infty} \tilde{\mathcal{D}}_{\infty} E ; \tilde{M}=\tilde{\rho}_{\infty} \tilde{\mathcal{D}}_{\infty} M$. Applying the boundary conditions $Z\left(\xi_{0}\right)=1$ and $Z \rightarrow 0$ as $\xi \rightarrow \infty$, we get:

$$
\frac{1}{2} \log \left(\frac{\left(\xi_{0}+1\right)}{\left(\xi_{0}-1\right)}\right)=\int_{0}^{1} \frac{\rho(Z) \mathcal{D}(Z) d Z}{E-M Z}
$$

These are general solutions for a prolate ellipsoid burning in a slowly moving (relative velocity) oxidizing environment in the fast reaction limit. The oxidizer flow does not have to be aligned with the axis of symmetry of the ellipsoidal particle, and the results are valid for any aspect ratio $\epsilon$. However, nothing has yet been said about the fuel flow rate. This information must be either prescribed, or a model of the condensed phase processes within the particle must be coupled to the above results.

\section{STEADY STATE SOLUTION FOR AN ABLATING SOLID}

In order to complete the solution a second relation between the parameters $E$ and $M$ must be found. This relationship comes from the set of equations and boundary conditions that describe the thermal degradation of the solid particle. The simplest case of physical interest is a vaporizing solid. Here, the net heat flux at any point on the particle surface $\dot{q}$ is related to the mass flux $\dot{m}$ by the effective heat of pyrolysis $\mathcal{L}$ by the relation $\dot{q}=\mathrm{L} \dot{m}$. Using the state relation between $\mathrm{Z}$ and $\tilde{T}$ given by Eq. 11 and using the fact that the velocity potential $\phi$ depends only on $\xi$, we can write:

$-\tilde{k} \frac{d \tilde{T}}{d Z} \frac{d Z}{d \xi}\left(\xi_{O}\right)=\left\llcorner\tilde{\rho} \tilde{\mathcal{D}}_{\infty} \frac{d \phi}{d \xi}\left(\xi_{O}\right)\right.$

Given that the Lewis number $\tilde{k} /\left(\tilde{\rho} \tilde{\mathcal{D}} C_{p}\right)=1$ is built into the state relations, the vaporizing solid boundary condition can be rewritten as:

$$
\mathcal{D}\left(\Delta H Y_{o}^{\infty}+C_{p}\left(\tilde{T}_{\infty}-\tilde{T}_{w}\right)\right) \frac{d Z}{d \xi}\left(\xi_{0}\right)=\mathrm{L} \frac{d \phi}{d \xi}\left(\xi_{0}\right)
$$

Using Eq. 21 and 22 to eliminate the derivatives, the following relation between $M$ and $E$ is obtained:

$$
M=\mathcal{B} /(1+\mathcal{B}) E
$$


Here, the parameter $\mathcal{B}$ is the Spalding "B number", defined as:

$\mathcal{B}=\left(\Delta H Y_{o}^{\infty}+C_{p}\left(\tilde{T}_{\infty}-\tilde{T}_{w}\right)\right) / \mathrm{L}$

Substitution of Eq. 30 into Eq. 27 yields the desired expression for E, and hence M.

$$
E=2\left(\log \left(\frac{\left(\xi_{0}+1\right)}{\left(\xi_{0}-1\right)}\right)\right)^{-1} \int_{0}^{1} \frac{\rho(Z) \mathcal{D}(Z) d Z}{1-(\mathcal{B} /(1+\mathcal{B})) Z}
$$

An explicit solution for $Z(\vec{r}, e, \mathcal{B})$ can be obtained for the particular case of $\rho \mathcal{D}=1$ [this assumption is reasonable because density and diffusivity have opposite relationship to temperature at constant pressure]. Under these circumstances, the integrals in equations (26) and (27) can be readily evaluated to yield:

$M\left(\xi_{0}, \mathcal{B}\right)=2\left(\log \left(\frac{\left(\xi_{0}+1\right)}{\left(\xi_{0}-1\right)}\right)\right)^{-1} \log (1+\mathcal{B})=\mathcal{B} /(1+\mathcal{B}) E$

$Z(\vec{r}, e, \mathcal{B})=\frac{(1+\mathcal{B})}{\mathcal{B}}(1-\exp (-W(\vec{r}, e, \mathcal{B})))$, Where:

$W(\vec{r}, e, \mathcal{B})=\log (1+\mathcal{B}) \log \left(\frac{(\xi+1)}{(\xi-1)}\right)\left(\log \left(\frac{\left(\xi_{0}+1\right)}{\left(\xi_{0}-1\right)}\right)\right)^{-1}$

A useful check of this result can be found by considering the limiting case of a spherical body in a stationary gas such as combustion of a liquid fuel droplet [10]. In the present case this corresponds to the limit $\mathrm{e} \rightarrow 1$. In this limit, $\xi_{0} \rightarrow \infty$, and since $\xi \geq \xi_{0}$, the same is true for $\xi$. Since $\xi_{0} \mathrm{c}=\mathrm{L} / 2=\mathrm{R}$, where $\mathrm{R}$ is the radius of the sphere, then for fixed $|\vec{r}|, \xi \rightarrow|\vec{r}| / c$. Thus:

$W \rightarrow \frac{R}{|\vec{r}|} \log (1+\mathcal{B}) ;$ solving for $Z$, we get:

$Z=\frac{(1+\mathcal{B})}{\mathcal{B}}\left(1-(1+\mathcal{B})^{-R /|\vec{r}|}\right)$

This differs from the result in $[10]$ by the prefactor $(1+\mathcal{B}) / \mathcal{B}$. The difference arises because the present study assumes $Z=1$ at the surface, while for a fuel droplet Liñán $\&$ Williams [10] regard $\mathcal{B}$ as a parameter to be determined by evaporative equilibrium and accept a discontinuity in $\mathrm{Z}$ at the droplet surface.

\section{TIME DEPENDENT EFFECTS - PARTICLE BURNOUT}

The analysis up to this point has assumed a steady state processes. However, there are two important exceptions that must be considered. First, the size of the particle is inherently time dependent because combustion reduces the mass of the particle. In principle, this process continues until the particle is entirely consumed, at which point the combustion terminates. The above analysis implicitly assumes that the time scale for mass loss is much slower than the time for a small parcel of fuel to be carried by advection or 
diffusion over a distance of the order of the semi-major axis of the ellipsoid. Under these conditions, the mass loss rate can be calculated as a steady-state process, and a global mass balance employed to determine the time history of the particle size. Second, for length scales far enough from the particle, the time scales are long enough for the species and energy transport to be unsteady and the combustion process may terminate before the steady state calculated above can be established in the far field. This means that the "outer expansion" describing the energy and species transport must be treated as inherently unsteady. Space precludes presenting this latter unsteady gas phase part of solution.

We first consider the consumption of the particle. The dimensional mass $M^{*}$ of a prolate ellipsoidal particle of density $\tilde{\rho}_{p}$ is:

$$
M^{*}=\frac{4 \pi}{3} \tilde{\rho}_{p}\left(\frac{L}{2}\right)^{3} e^{2}
$$

The mass loss rate can be determined from the source term in the far field velocity field as:

$$
\dot{M}=4 \pi c \tilde{M}=4 \pi \tilde{\rho}_{\infty} \tilde{\mathcal{D}}_{\infty}\left(\frac{L}{2}\right) \sqrt{1-\mathrm{e}^{2}} M\left(\xi_{0}, \mathcal{B}\right)
$$

Now introducing a dimensional time scale $\tilde{t}_{P}$, and a dimensionless time $\tau_{P}=\tilde{t} / \tilde{t}_{P}$ and the initial particle major axis length $L_{0}$ the particle mass balance equation becomes:

$$
\ell \frac{d \ell}{d \tau_{P}}=-1 ; \text { or: } \ell=\frac{L}{L_{0}}=\sqrt{1-2 \tilde{t} / \tilde{t}_{P}} ; \text { Where: } \tilde{t}_{P}=\left(\frac{L_{0}}{2}\right)^{2} \frac{1}{\tilde{\mathcal{D}}_{\infty}} \frac{\tilde{\rho}_{p}}{\tilde{\rho}_{\infty}} \frac{e^{2}}{\sqrt{1-\mathrm{e}^{2}}} \frac{1}{M\left(\xi_{0}, \mathcal{B}\right)}
$$

The particle burnout is inherently time dependent because vaporization reduces the size $L=1 L_{0}$ and the time scale for burnout is much longer than gas phase diffusion-convection time scale $\tilde{t}_{g}=(L / 2)^{2}\left(1 / \tilde{\mathcal{D}}_{\infty}\right)$. Particle burnout occurs at $\tilde{t}=\tilde{t}_{P} / 2$. Eq. 39 is the generalization of the " $d^{2}$ law" for fuel droplets. Note that the factor $\tilde{\rho}_{p} / \tilde{\rho}_{\infty} \sim 10^{3}$ appearing in the definition of $\tilde{t}_{P}$ means that the particle consumption time is much longer than the gas phase diffusion and advection time on the scale of the particle. This justifies the steady state analysis of particle burning. However, when there is small relative motion, the establishment of the steady state in the far field also takes a long time relative to the gas-phase diffusion time. The analysis of this competition between the two processes is not considered here. While not derived here, the transient far field solution for the prolate ellipsoid is given below in Eq. 41.

$$
Z \cong \frac{E L_{O}}{2|\vec{r}|} \sqrt{\left(1-\mathrm{e}^{2}\right)\left(1-2 \tilde{t} / \tilde{t}_{P}\right)}
$$

\section{Oblate Ellipsoids}

In this section we return to the study of the shape dependent combustion processes, this time for an oblate ellipsoid. Again, let $(\tilde{x}, \tilde{r})$ denote a cylindrical coordinate system aligned with the axis of symmetry of the ellipsoidal particle. Since the semi-major axis is by definition now in the radial direction, its length is denoted by $R$. The oblate ellipsoidal coordinate system $(\xi, \eta)$ is now defined as:

$$
\tilde{r}^{2}=c^{2}\left(1+\xi^{2}\right)\left(1-\eta^{2}\right) \text { and } \tilde{x}=c \xi \eta
$$


The quantity $c$ is redefined by requiring that $\xi=\xi_{0}$ gives an oblate ellipsoid of rotation with semi-major axis $c \sqrt{\left(1+\xi_{0}^{2}\right)}$ and semi-minor axis $\mathrm{c} \xi_{0}$. Thus:

$\frac{\tilde{r}^{2}}{c^{2}\left(1+\xi_{0}^{2}\right)}+\frac{\tilde{x}^{2}}{c^{2} \xi_{0}^{2}}=1$

Since the aspect ratio $\epsilon$ is the ratio of semi-minor to semi-major axes,

$\xi_{0}=e / \sqrt{1-e^{2}} \quad c=R \sqrt{1-e^{2}}$

Finally, the ellipsoidal coordinates can be written explicitly in terms of $(\tilde{x}, \tilde{r})$ as follows:

$\xi=\frac{1}{\sqrt{2} c}\left(\tilde{x}^{2}+\tilde{r}^{2}-c^{2}+\sqrt{\left(\tilde{x}^{2}+\tilde{r}^{2}-c^{2}\right)^{2}+(2 \tilde{x} c)^{2}}\right)^{1 / 2} \quad \eta=\frac{\tilde{x}}{c \xi}$

The coordinate geometry for contours of constant $\xi$ starting at the particle surface is shown for an ellipsoid of aspect ratio $\mathrm{e}=1 / 9$ to 1 in Fig. 1 .

Writing the conservation Eq. 5 and 6 in oblate ellipsoidal coordinates and assuming that $\tilde{\rho}, \tilde{\phi}$ and $Z$ are functions of $\xi$ only, we get:

$\frac{d}{d \xi}\left(\left(1+\xi^{2}\right) \tilde{\rho}(\xi) \frac{d \tilde{\phi}}{d \xi}\right)=0$

$\frac{d}{d \xi}\left[\left(1+\xi^{2}\right) \tilde{\rho}(\xi) Z(\xi) \frac{d \tilde{\phi}}{d \xi}-\tilde{\rho}(\xi) \tilde{\mathcal{D}}(\xi)\left(1+\xi^{2}\right) \frac{d Z}{d \xi}\right]=0$

Once again, these equations can be readily integrated to yield:

$$
\left(1+\xi^{2}\right) \tilde{\rho}(\xi) \frac{d \tilde{\phi}}{d \xi}(\xi)=\tilde{M}_{O}
$$

$$
\tilde{M}_{O} Z(\xi)-\tilde{\rho}(\xi) \tilde{\mathcal{D}}(\xi)\left(1+\xi^{2}\right) \frac{d Z}{d \xi}=\tilde{E}_{O}
$$

Integrating Eq. 48 again and imposing the boundary condition that $\mathrm{Z}=1$ at the particle surface $\xi=\xi_{\mathrm{O}}$ yields the result:

$$
\int_{Z}^{1} \frac{\tilde{\rho}(Z) \tilde{\mathcal{D}}(Z) d Z}{\tilde{E}_{O}-\tilde{M}_{O} Z}=\arctan (\xi)-\arctan \left(\xi_{0}\right)
$$

Far from the particle, as $\xi \rightarrow \infty$, we must have $Z \rightarrow 0$. Thus:

$$
\int_{0}^{1} \frac{\tilde{\rho}(Z) \tilde{\mathcal{D}}(Z) d Z}{\tilde{E}_{O}-\tilde{M}_{O} Z}=\frac{\pi}{2}-\arctan \left(\xi_{0}\right)
$$


This yields one relation between $\tilde{M}_{O}$ and $E_{O}$. It also permits Eq. 49 to be rewritten in the form:

$\int_{0}^{Z} \frac{\tilde{\rho}(Z) \tilde{\mathcal{D}}(Z) d Z}{\tilde{E}_{O}-\tilde{M}_{O} Z}=\frac{\pi}{2}-\arctan (\xi)$

For the Oblate ellipsoid, when $|\vec{r}| / R \gg 1, \xi \gg 1$, and $\xi \cong|\vec{r}| / c$. Thus, far from the particle, the solution for $\mathrm{Z}$ reduces to:

$Z \simeq \tilde{E}_{O} /\left(\tilde{\rho}_{\infty} \tilde{\mathcal{D}}_{\infty} \xi\right)=\tilde{E}_{O} c /\left(\tilde{\rho}_{\infty} \tilde{\mathcal{D}}_{\infty}|\vec{r}|\right)$

The similarities of these results with those obtained earlier for the prolate ellipsoid are obvious. This similarity extends to the determination of the parameters $\tilde{E}_{O}$ and $\tilde{M}_{O}$. Introducing dimensionless quantities, for $E_{O}$ and $M_{O}, \tilde{E}_{O}=\tilde{\rho}_{\infty} \tilde{D}_{\infty} E_{O} \& \tilde{M}_{O}=\tilde{\rho}_{\infty} \tilde{D}_{\infty} M_{O}$. The required second relation between these parameters is identical with the result for the prolate ellipsoid.

$M_{O}=\mathcal{B} /(1+\mathcal{B}) E_{O}$

This must be true on physical grounds, since the ablation boundary condition is entirely locally determined. It can also be derived mathematically; the steps are identical with those used for the prolate ellipsoid. Using Eq. 54 in Eq. 51, the solution for $\mathrm{E}_{\mathrm{O}}$ is readily obtained.

$$
E_{O}=\int_{0}^{1} \frac{\rho(Z) \mathcal{D}(Z) d Z}{1-(\mathcal{B} /(1+\mathcal{B})) Z} /\left(\frac{\pi}{2}-\arctan \left(\xi_{0}\right)\right)
$$

For the special case of $\rho \mathcal{D}=1$, the integral can be evaluated with the result:

$$
\begin{aligned}
& E_{O}=\frac{(1+\mathcal{B})}{\mathcal{B}} \log (1+\mathcal{B}) /\left(\frac{\pi}{2}-\arctan \left(\xi_{0}\right)\right) \\
& M_{O}=\log (1+\mathcal{B}) /\left(\frac{\pi}{2}-\arctan \left(\xi_{0}\right)\right)
\end{aligned}
$$

The corresponding solution for the Mixture fraction $Z\left(\xi, \xi_{0}, \mathcal{B}\right)$ is:

$$
\begin{aligned}
& Z\left(\xi, \xi_{0}, \mathcal{B}\right)=\frac{(1+\mathcal{B})}{\mathcal{B}}\left(1-\exp \left(-W\left(\xi, \xi_{0}, \mathcal{B}\right)\right)\right), \text { Where: } \\
& W\left(\xi, \xi_{0}, \mathcal{B}\right)=\log (1+\mathcal{B})\left(\frac{\pi}{2}-\arctan (\xi)\right) /\left(\frac{\pi}{2}-\arctan \left(\xi_{0}\right)\right)
\end{aligned}
$$

We now consider the time evolution of the burning rate for the oblate ellipsoid. The dimensional mass of an oblate ellipsoid particle of radius $\mathrm{R}$, aspect ratio $\epsilon$, and density $\tilde{\rho}_{p}$ is: 
$M^{*}=\frac{4 \pi}{3} \tilde{\rho}_{p} e R^{3}$

The dimensional mass loss rate through a sphere far from the particle $\dot{M}$ is:

$\dot{M}=4 \pi \tilde{\rho}_{\infty} \tilde{\mathcal{D}}_{\infty} R \sqrt{1-\mathrm{e}^{2}} M_{O}\left(\xi_{0}, \mathcal{B}\right)$

Equating the rate of change of the particle mass with minus the mass flux far from the particle, the time history of the particle radius can be expressed in terms of the initial radius $R_{O}$ and the oblate particle dimensional time scale $t_{O}$ as:

$$
\begin{aligned}
& \frac{d M^{*}}{d \tilde{t}}=-\dot{M} ; \text { this gives: } \frac{R}{R_{O}}=\sqrt{1-2 \tilde{t} / \tilde{t}_{O}} ; \text { Where, } \\
& \tilde{t}_{O}=\frac{R_{O}^{2}}{\tilde{\mathcal{D}}_{\infty}} \frac{\tilde{\rho}_{p}}{\tilde{\rho}_{\infty}} \frac{e}{\sqrt{1-\mathrm{e}^{2}}} \frac{1}{M_{O}\left(\xi_{0}, \mathcal{B}\right)}
\end{aligned}
$$

The transient far field solution for the oblate ellipsoid can be found immediately. Equation (41) shows the far field limit of the solution for the prolate ellipsoid. This solution applies to the oblate case if $E$ is replaced by $E_{O}, L_{O} / 2$ replaced by $R_{O}$, and $\tilde{t}_{P}$ is replaced by $\tilde{t}_{O}$. All the far-field solutions for the prolate ellipsoid also apply to the oblate ellipsoid using these substitutions. The particle burnout reduces $R$ and it occurs at $\tilde{t}=\tilde{t}_{O} / 2$.

\section{Burnout time for different size and aspect ratio prolate and oblate firebrands}

Figure 3 shows the computations of particle burnout times using Eqs. 40 and 63. In these equations, three different $\mathcal{B}$ numbers are used; 0.1 to represent glowing combustion of wood char, 0.6 to represent flaming combustion of wood, and 1.2 given by reference [4]. Wood firebrands are assumed to have a density of 250 $\mathrm{kg} / \mathrm{m}^{3}$ [4]. The logic for selecting these $\mathcal{B}$ numbers is as follows: For forest fires we are primarily concerned with flaming or glowing combustion of wood and wood char. For wood char, as evidenced from data [11, 12] both reactions $\mathrm{C}+\mathrm{O}_{2} \rightarrow \mathrm{CO}_{2}$ and $2 \mathrm{C}+\mathrm{O}_{2} \rightarrow 2 \mathrm{CO}$ take place at the surface. The question is how much of which reaction occurs because it depends on the surface temperature of glowing char (1254K [11]). At higher temperatures, experiments $[13,14]$ show a thin, bluish, CO flame separated from the carbon surface. However, this mechanism is not likely for firebrands. Thus, to estimate the B-number for glowing combustion of wood char, Evans [11] data is used. The estimated B number is $\cong 0.1$. Recent work of Rangwala [15] shows that the average B-number for Douglas fir wood is $\cong 0.6$. Surprisingly, Fig. 3 shows that the firebrand burnout time is shape independent. The burnout time depends only on the firebrand mass under the assumptions used.

\section{A BRIEF NOTE ON VELOCITY FIELD}

The velocities induced by the combustion of the particle surface can also be calculated. Since the velocity potential $\tilde{\phi}$ depends only on the appropriate (prolate or oblate) ellipsoidal coordinate $\xi$, the velocity always points in the direction of the unit normal $\vec{i}_{\xi}$. Thus, for a prolate ellipsoid:

$$
\vec{u}=\vec{i}_{\xi} \tilde{V}_{\xi} ; \text { where: } \tilde{V}_{\xi}=\frac{\mathcal{D}_{\infty}}{L} \frac{2}{\sqrt{1-\mathrm{e}^{2}}} \frac{M}{\rho(Z)} \frac{1}{\sqrt{\left(\xi^{2}-1\right)\left(\xi^{2}-\eta^{2}\right)}}
$$


This is the general solution for the velocity field for any prolate ellipsoid regardless of the state relation defining $\rho(Z)$ used. An interesting property of this solution is the increasing value of the velocity near the ends of the particle as $\epsilon \rightarrow 0$. This can be readily seen by noting that on the particle surface, $\xi=\xi_{0}, \eta=\tilde{x} /(L / 2)$. The velocity approaches a square root singularity at each end as the particle shape becomes more like that of a needle. The analysis and results for an oblate ellipsoid are similar.

\section{CONCLUSIONS}

In this work the burning rate for a class of particles ranging from a thin needle to a sphere (prolate ellipsoids) and from a sphere to a thin disc (oblate ellipsoids) is studied using methods based directly on the underlying conservation equations of fluid mechanics and combustion science. These shapes of particles are reasonable representation of various (unknown) shapes of firebrands lofted by forest fires. The formulae obtained are simple enough to be evaluated without elaborate numerical computations.

The analysis assumes "quasi-steady" burning. The Reynolds number based on the over-all particle dimension and velocity relative to the ambient atmosphere is assumed to be small. The gas phase combustion processes are represented by the evolution of a mixture fraction variable. It is shown that the velocity field near the particle can be described by a potential flow whose functional form is determined by the mass conservation equation and that this flow satisfies the particle surface boundary conditions. Gas phase solutions are obtained for two-parameter family of firebrand shapes composed of oblate and prolate ellipsoids of revolution. The ambient velocity field does not need to be aligned with the particle axis of symmetry, so that the composite velocity and mixture fraction fields are three-dimensional. While a variety of steady-state condensed phase models are compatible with this analysis, results are presented for a simple physically interesting case of an ablating solid describable by the Spalding $\mathcal{B}$-number. $\mathcal{B}$-numbers representative of wood and wood char are used and all quantities are calculated as a function of ellipsoidal aspect ratio, $\mathcal{B}$ number, and the Reynolds number. A surprising result is found: the firebrand burnout time is shape independent and the burnout time depends only on the firebrand mass under the assumptions used.

\section{REFERENCES}

[1] Tarifa C. S., del Notario P. P., Moreno F. G., "On flight paths and lifetimes of burning particles of wood," Proceedings of the Tenth International Symposium on Combustion, 1965, pp. 1021-1037.

[2] Albini F. A., "Transport of firebrands by line thermals," Combustion Science and Technology 32, 1983, 277-288. doi:10.1080/00102208308923662.

[3] Manzello S. L, Shields J. R, Hayashi Y, Nii D, "Investigating the vulnerabilities of structures to ignition from a firebrand attack," Proceedings of the Ninth International Symposium on Fire Safety Science, 2008, 21-26, pp. 143-154.

[4] Koo E, Linn R. R, Pagni P. J, "Modeling firebrand transport in wildfires using HIGRAD/ FIRETEC," International Journal of Wildland Fire, 2012, 21, 396-417.

[5] Woycheese J. P, Pagni P. J, Liepmann D, "Brand propagation from large-scale fires. Journal of Fire Protection Engineering 10, 32-44. 1999. doi:10.1177/104239159901000203.

[6] Sardoy N, Consalvi J. L, Porterie B, Fernandez-Pello A. C, "Modeling transport and combustion of firebrands from burning trees. Combustion and Flame 150, 151-169. 2007.

[7] Koo E, Pagni P. J, Weise D. R, Woycheese J. P, "Firebrand and spotting ignition in large-scale fires," International Journal of Wildland Fire, 2010, 19, 818-843.

[8] Goldstein, S. “Lectures on Fluid Mechanics,” Interscience, New York, p. 89, 1960.

[9] Magnus, W. and Oberhettinger, F., "Formulas and Theorems for the Functions of Mathematical Physics," Chelsea, New York. p. 149, 1943.

[10] Liñán A. and Williams, F.A., Fundamental Aspects of Combustion, Oxford Univ. Press, Oxford, pp. 74-78, 1993. 
[11] Evans, D. D. and H. W. Emmons. "Combustion of wood charcoal." Fire Safety Journal 1.1 (1977): 57-66.

[12] Blackshear, P. L., and K. A. Murty. "Heat and mass transfer to, from, and within cellulosic solids burning in air." Symposium (International) on Combustion. Vol. 10. No. 1. Elsevier, 1965.

[13] Davis, H., and H. C. Hottel. "Combustion Rate of Carbon-Combustion at a Surface Overlaid with Stagnant Gas." Industrial \& Engineering Chemistry 26.8 (1934): 889-892.

[14] Tu, C. M., H. Davis, and H. C. Hottel. "Combustion Rate of Carbon-Combustion of Spheres in Flowing Gas Streams." Industrial \& Engineering Chemistry 26.7 (1934): 749-757.

[15] Rangwala, Ali S., "Flame spread Analysis using a Variable B-Number," Ph. D. thesis, UC San Diego, 2006. 


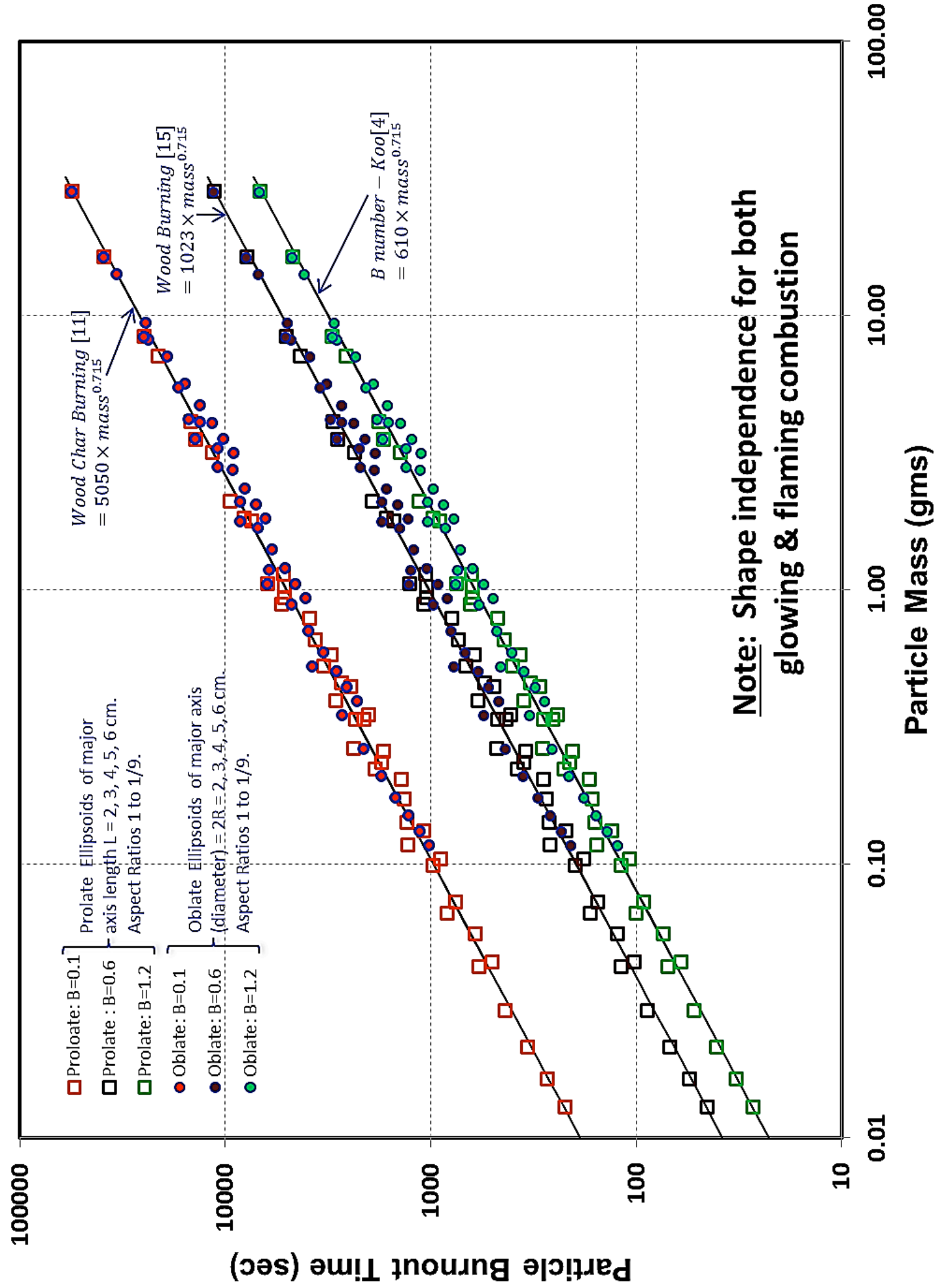

Fig. 3: Particle burnout time for Prolate and Oblate ellipsoids, with major axis ranging from 2 to $6 \mathrm{~cm}$, and aspect ratio ' $\epsilon$ ' ranging from 1 to $1 / 9$. Note that ellipsoids with $\epsilon=1$ are spherical and have the largest mass. Calculations for three B-numbers are plotted: $\mathrm{B}=0.1,0.6 \& 1.2$. They correspond to glowing char and flaming wood combustion. 